\title{
SUPERSONIC INVERSE DESIGN METHOD FOR WING-FUSELAGE DESIGN
}

\author{
Shinkyu Jeong, Shigeru Obayashi and Kazuhiro Nakahashi \\ Tohoku University, 980-77, Sendai, Japan \\ Toshiyuki Iwamiya \\ National Aerospace Laboratory, Chofu, Tokyo 182, Japan \\ Kisa Matsushima \\ Fujitsu Limited, Chiba 261, Japan
}

\begin{abstract}
An inverse method for supersonic wing design has been developed. The present method is based on iterative "residual-correction" concept. The geometry correction is calculated by solving linearized small perturbation (LSP) equation with a difference of surface pressure distributions as a boundary condition. In the present method, LSP equation is analytically transformed to integrodifferential equations by using Green's theorem. Design results of an isolated wing and wing-fuselage configuration are presented here.
\end{abstract}

\section{KEYWORDS}

Supersonic inverse design, residual-correction concept, Green’s theorem, SST

\section{INTRODUCTION}

With a recent trend of the worldwide growth of air transportation, development of a next generation supersonic transport (SST) is under consideration in the United States, Europe, and Japan. There have been a few supersonic transport so far, such as, the TU-144 developed by the Soviet Union and the Concorde by the joint of the UK and France. But the TU-144 ceased its regular operation some time before 1985 because of problems with the engines and wing design. Unlike the TU-144, the Concorde continues its commercial service today. From the technological point of view, the Concorde is a great achievement. However, from the economical point of view, the Concorde cannot be said a success. One of its shortcomings is low-speed performance $(\mathrm{L} / \mathrm{D}=4)$. Its poor $\mathrm{L} / \mathrm{D}$ has to be compensated by high fuel burn, which increases the operating cost. To guarantee economic success of the next generation SST, 
a higher $\mathrm{L} / \mathrm{D}$ ratio is indispensable. To achieve this goal, a new design technique for supersonic wings is necessary. There were several design methods $[1,2,3]$ used for supersonic wings. Most of these methods consider only warp optimization by using the linearized method, however, it is also important to consider wing thickness control to obtain a wing of high performance. Especially, the thickness control plays an important role, such as, for prevention of the leading-edge separation and for laminar flow control.

In this study, a three-dimensional supersonic wing design method that can determine both the warp and thickness at the same time is developed. The present method is extended from Takanashi's inverse design method [4] used for the transonic wing design. Takanashi solved the inverse problem by using the integral form of the transonic small perturbation equation with "residual-correction" concept. This paper will discuss the mathematical formulation of the present method and show two design results. One is for an isolated-wing configuration and the other is for a wing-fuselage combination that is the baseline design of National Aerospace Laboratory's experimental scaled SST.

\section{DESIGN PROCEDURE}

The inverse problem in the aerodynamic shape design is to find a geometry that yields a specified surface pressure distribution. The procedure of finding a corresponding geometry in the present method is described as follows. First, a target pressure distribution and an initial geometry are inputted to a design system and then the surface pressure distribution of this initial geometry is obtained by the flow analysis. In this design system, inverse calculation stage is separated from the flow analysis stage. Thus the any type of analysis, even an experiment, can be used for the flow analysis tool. In this study, the Euler/Navier-Stokes solver is used for the flow analysis. From the computed and target pressure distributions, the pressure difference is calculated. Using this pressure difference as a boundary condition, a geometry correction is obtained by solving the linearized small perturbation (LSP) equation. By modifying the initial geometry with the geometry correction, a new geometry is produced. This process will be iterated until $\Delta$ Cp becomes sufficiently small. Figure 1 shows the flowchart of the present inverse design procedure.

\section{INTEGRAL FORMULATION OF INVERSE METHOD}

In a supersonic flow, the small perturbation potential equation can be expressed in the linearized form as

$$
\left(M_{\infty}^{2}-1\right) \bar{\phi}_{\overline{x x}}-\bar{\phi}_{\overline{y y}}-\bar{\phi}_{\overline{z z}}=0
$$


And a pressure coefficient on wing surface and a tangency condition can be written as

$$
\begin{aligned}
C_{p \pm}(\bar{x}, \bar{y}) & =-2 \bar{\phi}_{\bar{x}}(\bar{x}, \bar{y}, \pm 0) \\
\frac{\partial \bar{f}_{ \pm}(\bar{x}, \bar{y})}{\partial \bar{x}} & =\bar{\phi}_{\bar{z}}(\bar{x}, \bar{y}, \pm 0)
\end{aligned}
$$

where the subscript ' \pm ' denote the upper and lower surface of geometry. For brevity, a Prandtl-Grauert transformation is performed and new coordinates denoted with $x, y, z$ are introduced. The transformed equations are written as

$$
\begin{aligned}
& \phi_{x x}-\phi_{y y}-\phi_{z z}=0 \\
& C_{p \pm}\left(x, \frac{y}{\beta}\right)=-2 \beta^{2} \phi_{x}(x, y, \pm 0) \\
& \frac{\partial_{ \pm}(x, y)}{\partial x}=\beta^{3} \phi_{z}(x, y, \pm 0)
\end{aligned}
$$

The objective of the inverse problem is to find the corresponding geometry that realizes the specified pressure distribution. The most common approach is to solve the partial difference equation (4) with the boundary condition, Eq. (5). But finding an exact geometry at one time is very difficult. In this paper, to develop a more general design procedure with a wide range of application, an iterative "residual-correction" concept is introduced. Suppose the solution of Eq. (4), $\phi(x, y, z)$, for the initial geometry $f(x, y)$ is given and the perturbation, $\Delta \phi(x, y, z)$, is occurred to this known flow. After some algebraic manipulations, the governing equation and boundary conditions of this method become

$$
\begin{aligned}
& \Delta \phi_{x x}-\Delta \phi_{y y}-\Delta \phi_{z z}=0 \\
& \Delta C_{p \pm}\left(x, \frac{y}{\beta}\right)=-2 \beta^{2} \Delta \phi_{x}(x, y, \pm 0) \\
& \frac{\Delta \partial_{ \pm}(x, y)}{\partial x}=\beta^{3} \Delta \phi_{z}(x, y, \pm 0)
\end{aligned}
$$

However, the governing equation (7) is still field expression. It requires many endeavors to solve this P.D.E directly. In the present method, this difficulty is eliminated by applying Green's theorem [5,6] to Eq. (7). The solution of governing equation (7), $\Delta \phi(x, y, z)$, is expressed in the integrodifferntial form as

$$
\begin{aligned}
\Delta \phi(x, y, z)=- & \frac{1}{2 \pi} \frac{\partial}{\partial x} \iint_{\tau_{1}}\left[\left(\Delta \phi_{\zeta}(\xi, \eta,+0)-\Delta \phi_{\zeta}(\xi, \eta,-0)\right) \times \varphi(x, y, z ; \xi, \eta, 0)\right] d \xi d \eta \\
& \frac{1}{2 \pi} \frac{\partial}{\partial x} \iint_{\tau_{1}}\left[(\Delta \phi(\xi, \eta,+0)-\Delta \phi(\xi, \eta,-0)) \times \varphi_{\zeta}(x, y, z ; \xi, \eta, 0)\right] d \xi d \eta
\end{aligned}
$$

where 


$$
\varphi(x, y, z ; \xi, \eta, \zeta)=\cosh ^{-1} \frac{x-\xi}{\sqrt{(y-\eta)^{2}+(z-\zeta)^{2}}}
$$

The integral surface $\tau_{1}$ is the part of $z=0$ plane contained within the Mach forecone from the point $(x, y, z)$, that is, the area bounded by the line $\xi=-\infty$ and the hyperbola;

$$
(x-\xi)^{2}-(y-\eta)^{2}-(z-\zeta)^{2}=0
$$

Namely, the integral surface $\tau_{1}$ is inside of the Mach forecone on the planform. The integral surface $\tau_{1}$ the triangular planform is shown in Fig. 2.

To utilize the pressure distributions as a boundary condition, Eq. (10) is differentiated with respect to $x$ and by adding the values of the resulting $\Delta \phi_{x}(x, y, z)$ at $z=+0$ and $z=-0$ we obtain

$$
\begin{gathered}
\Delta u_{s}(x, y)=-\Delta w_{s}(x, y)+\frac{1}{\pi} \iint_{\tau_{1}} \frac{(x-\xi) \Delta w_{s}(\xi, \eta)}{{\sqrt{(x-\xi)^{2}-(y-\eta)^{2}}}^{3}} d \xi d \eta \\
\Delta u_{s}(x, y)=\Delta \phi_{x}(x, y,+0)+\Delta \phi_{x}(x, y,-0) \\
\Delta w_{s}(x, y)=\Delta \phi_{z}(x, y,+0)-\Delta \phi_{z}(x, y,-0)
\end{gathered}
$$

Similarly, differentiating both sides of Eq. (10) with respect to $z$ and adding resulting $\Delta \phi(x, y, z)_{z}$ at $z=+0$ and $z=-0$, we obtain

$$
\begin{gathered}
\Delta w_{a}(x, y)=-\Delta u_{a}(x, y)+\frac{1}{\pi} \iint_{\tau_{1}} \frac{(x-\xi) \Delta u_{a}(\xi, \eta)}{(y-\eta)^{2} \sqrt{(x-\xi)^{2}-(y-\eta)^{2}}} d \xi d \eta \\
\Delta u_{a}(x, y)=\Delta \phi_{x}(x, y,+0)-\Delta \phi_{x}(x, y,-0) \\
\Delta w_{a}(x, y)=\Delta \phi_{z}(x, y,+0)-\Delta \phi_{z}(x, y,-0)
\end{gathered}
$$

By solving Eqs. (13) and (16) with the boundary conditions $\Delta u_{s}$ and $\Delta u_{a}$, the geometry terms $\Delta w_{s}$ and $\Delta w_{a}$ can be obtained. As defined in Eqs. (15) and (18), $\Delta w_{s}(x, y)$ and $\Delta w_{a}(x, y)$ represent the derivatives of thickness and camber correction, respectively.

The integrated value for the thickness correction, however, does not guarantee to satisfy the closure condition at trailing edge. In this paper, $\Delta w_{s}$ are modified to satisfy the closure condition. Modifications are performed according to the following equation:

$$
\Delta w_{s}^{\bmod }(x, y)=\Delta w_{s}(x, y)-\frac{\int_{L . E}^{T . E .} \Delta w_{s}(\xi, y) d \xi}{\overline{d x}} \times \frac{d x}{l}
$$

where $l$ is local chord length and $\overline{d x}$ is chord length divided by number of panels at each spanwise location. 
The geometry correction can be computed by performing the numerical integration in the $x$ direction.

$$
\Delta f_{ \pm}(x, y)=\frac{1}{2} \int_{L . E .}^{x} \Delta w_{a}(\xi, y) d \xi \pm \frac{1}{2} \int_{L . E .}^{x} \Delta w_{s}^{\bmod }(\xi, y) d \xi
$$

This equation may be contrasted with the camber design of Ref. 7.

Since Eqs. (13) and (16) are based on LSP equation, they become invalid where the vorticity is generated. Such regions are typically around the root and tip of the wing. At the wing root, the bilateral symmetry is usually assumed for the flow analysis, but $\frac{\partial p}{\partial y} \neq 0$ when the straight isobar pattern is enforced for prescribing a pressure distribution for a swept wing. This breaks the irrotational flow assumption at the wing root. At the wing tip, on the other hand, the flow is naturally rotational due to the wing tip vortex. Therefore, the Eqs. (13) and (16) should be replaced with lower order approximations in those regions as

$$
\begin{aligned}
& \Delta u_{s}(x, y)=-\Delta w_{s}(x, y) \\
& \Delta w_{a}(x, y)=-\Delta u_{a}(x, y)
\end{aligned}
$$

These equations are now the supersonic linearized pressure coefficient equations.

\section{DESIGN RESULTS}

\section{Isolated-wing configuration}

The planform used in this three-dimensional wing design problem is shown in Fig. 3. This planform is the so-called 0-th baseline configuration of the NAL SST program. The leading-edge sweep angle is about $70^{\circ}$ and the leading- and trailing-edge kinks are located at $43.96 \%$ and $40 \%$ spanwise sections, respectively. For inverse design, there are 50 panels in streamwise direction and 67 panels in spanwise direction. The target pressure distribution is obtained from the untwisted wing based on NACA-1204 airfoil in an inviscid flow at $\mathrm{M}=2.0$, $\alpha=2^{\circ}$. The initial wing is obtained from NACA-0003 airfoil.

Figure 4 shows comparison of surface pressure distributions at six spanwise sections among the target, initial, and designed wings after 6 design iterations. As shown in the figures, surface pressure distributions of designed wing coincide with those of target wing at all spanwise sections.

\section{Wing-fuselage combination}

The planform used in this wing-fuselage combination design problem is shown in Fig. 5. This planform is the 3-rd baseline configuration of the NAL SST program. For the inverse computation, the half span of wing is divide into 82 panels in spanwise direction and 50 panels in chordwise direction. Target pressure distribution is specified to have the elliptical load 
distribution to minimize the induced drag. The upper surface of target pressure distribution is designated to keep the laminar boundary layer as long as possible. Figure 6 shows comparison of surface pressure distributions at four spanwise sections among the target, initial, and designed wings after 8 design iterations. Though pressure distributions of designed wing doesn't perfectly coincide with target pressure distributions, they become very close to target pressure distributions. In Fig. 7, the spanwise load distribution of target, initial and designed wings are plotted. As seen in Fig. 7. the load distribution of designed wing coincides with that of target almost perfectly.

\section{CONCLUSION \& FUTURE WORK}

In this study, Takanashi's transonic inverse design method is extended to supersonic wing-fuselage design. Once target pressure distributions are specified, the corresponding wing configuration can be found by the present inverse design method with Euler/Navier-Stokes solvers. Design results confirmed the validity of this method.

To use this design method effectively, the target pressure distribution must be selected carefully. All design objectives should be translated into the target pressure distributions. The determination of an optimal target pressure distribution [8] will be studied in future for the supersonic cases.

\section{REFERENCES}

1. Carlson, H. W. and Miller, D. S., Numerical Methods for the Design and Analysis of Wings at Supersonic Speed, NASA TN D-7713, 1974.

2. Yoshida, K. and Hayama, K., Experimental and Numerical Study of Aerodynamic Characteristics for Second generation SST, SAE paper 91-2056, 1991.

3. K. B. Walkley, and G. E. Smith, Application of a Full Potential Method to Supersonic Aircraft Design and Analysis, Journal of Aircraft, Vol. 26, No. 1, pp.6-12, Jan., 1989.

4. Takanashi, S., Iterative Three-Dimensional Transonic Wing Design Using Integral Equations, Journal of aircraft, Vol. 22, No. 8, pp.655-660, Aug. 1985.

5. Heaslet, M. A., Lomax, H., and Jones, A. L., Volterra's Solution of the Wave Equation as Applied to Three-Dimensional Supersonic Airfoil Problem, NACA Rep. 889, 1947 (Formerly NACA TN 1412).

6. Lomax, H., Heaslet, M. A. and Franklyn, B. Fuller, Integrals and Integral Equation in Linearized Wing Theory, NACA Rep. 1054, 1951.

7. Carlson, H. W. and Middleton, W. D., A Numerical Method for Design of Camber Surfaces of Supersonic Wings with Arbitrary Planforms, NASA TN D-2341, 1964.

8. Obayashi, S., Jeong, S. and Matsuo, Y., New Blunt Trailing-Edge Airfoil Designed by Inverse Optimization Method, Journal of Aircraft, Vol.34, No.2, pp.255-257, March-April, 
1997. 


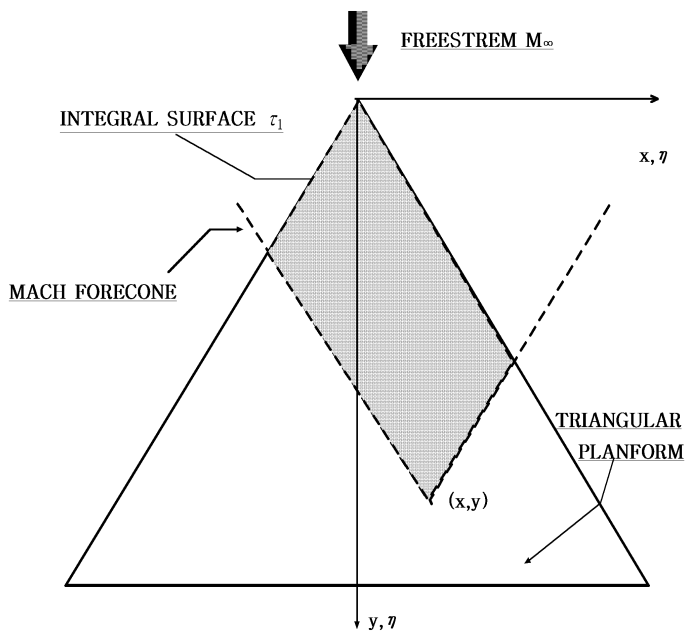

Fig. 1. Flowchrat of inverse design procedure

Fig. 2. Integral surface on the triangular planform

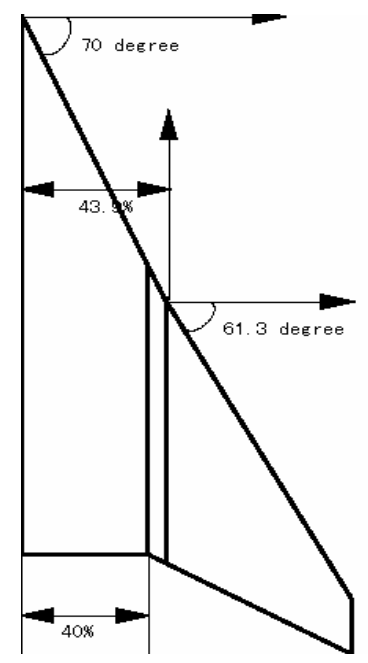

Fig. 3. Planform of wing-only configuration
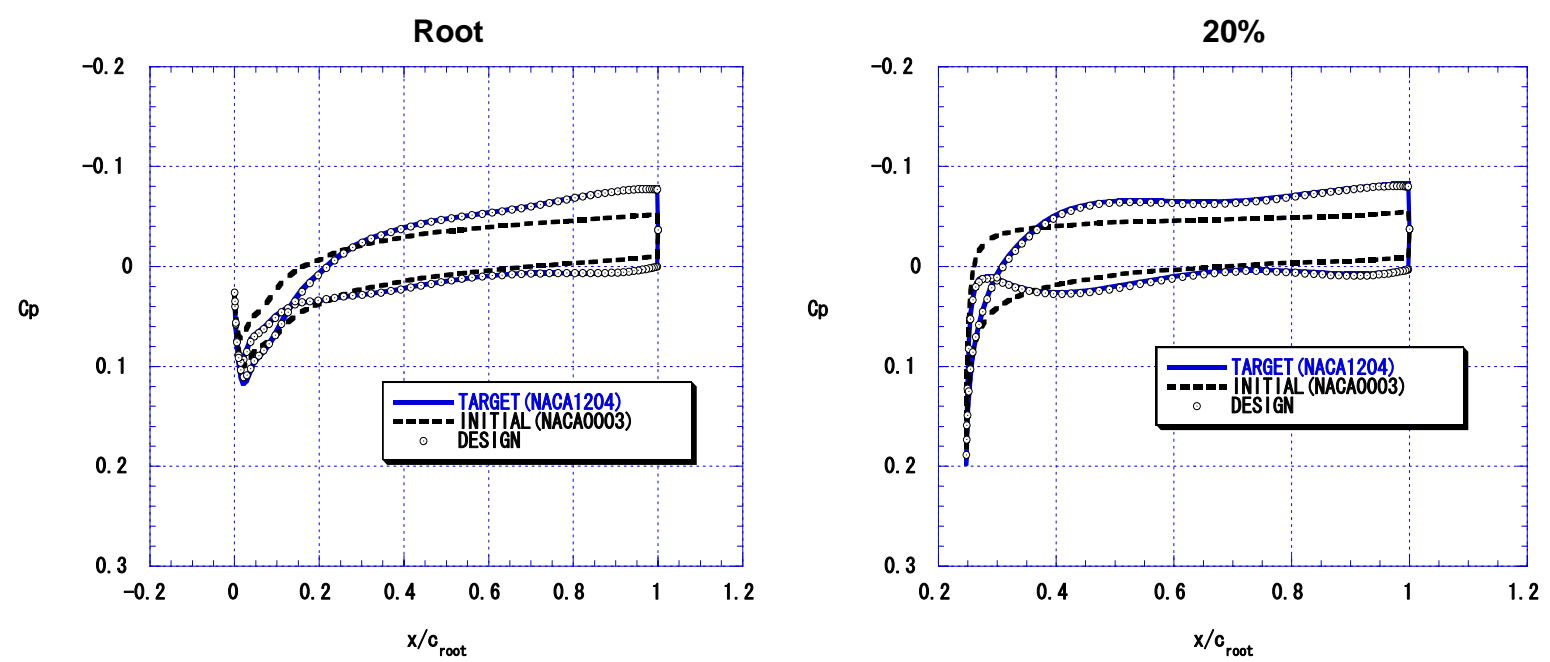

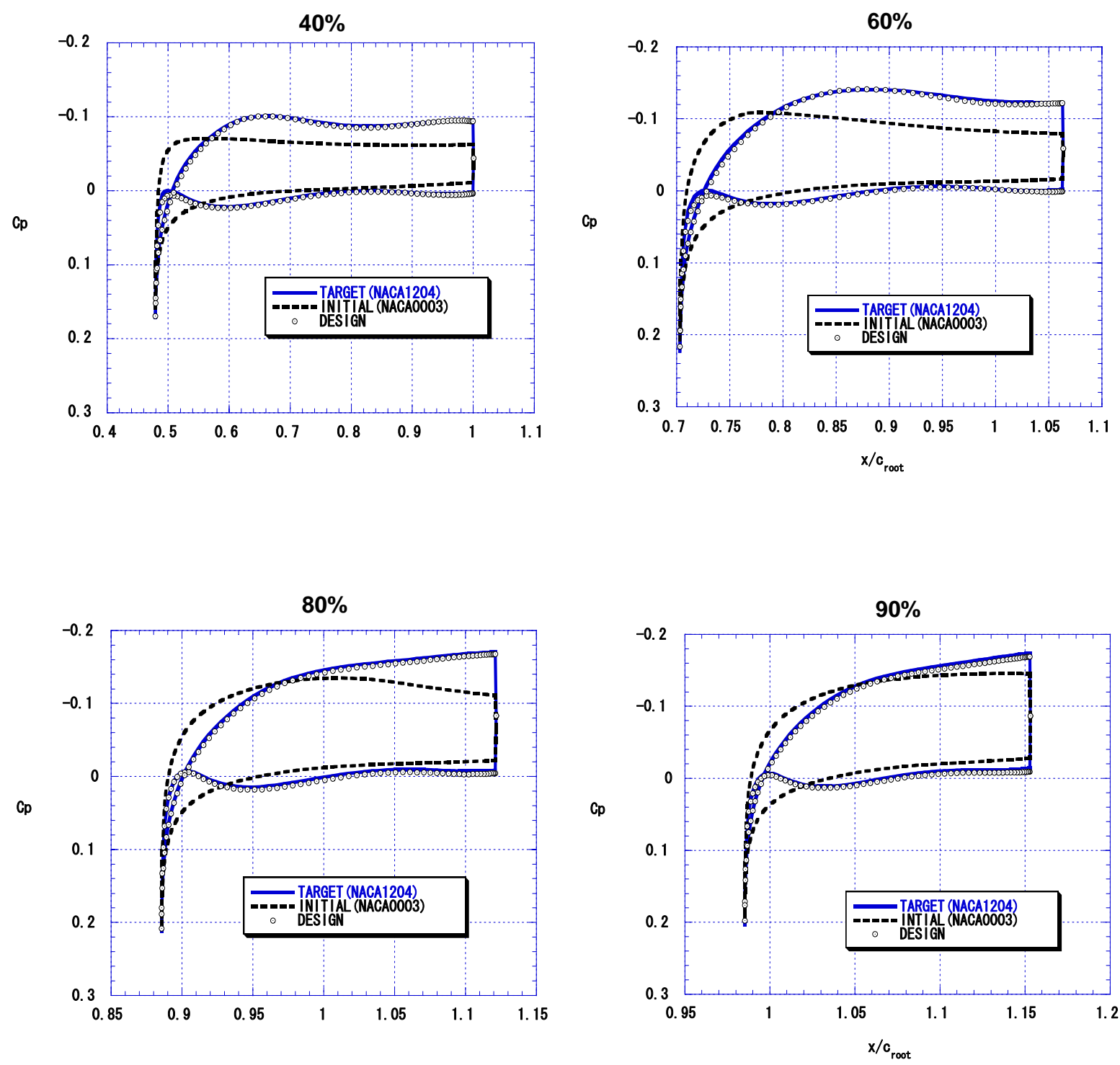

Fig. 4. Comparisons of surface pressure distributions among the target, initial, and designed wing in an isolated-wing configuration design problem

Fig. 5. Planform of wing-fuselage combination design 

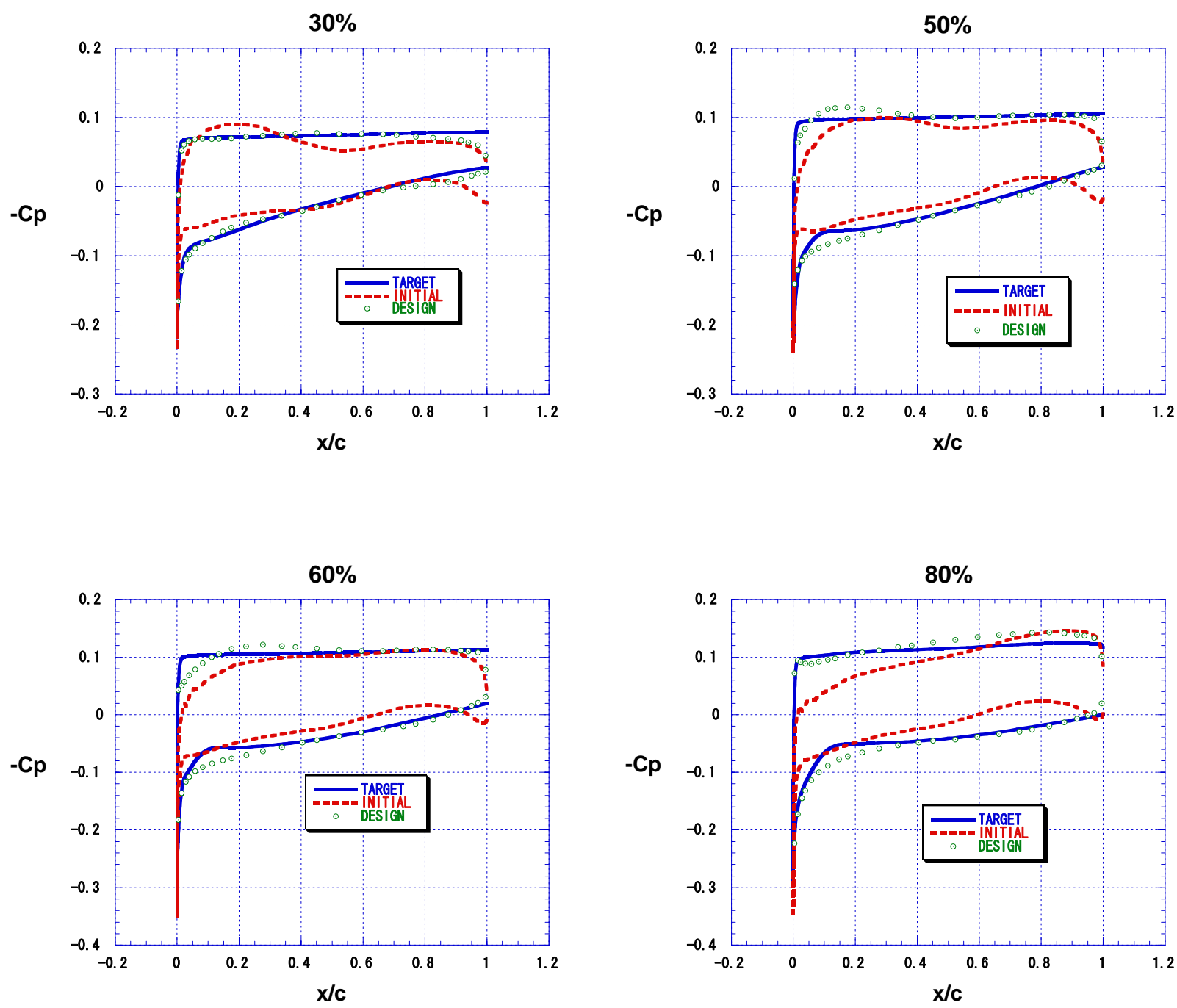

Fig. 6. Comparisons of surface pressure distributions among the target, initial, and designed wing in a wing-fuselage combination design problem

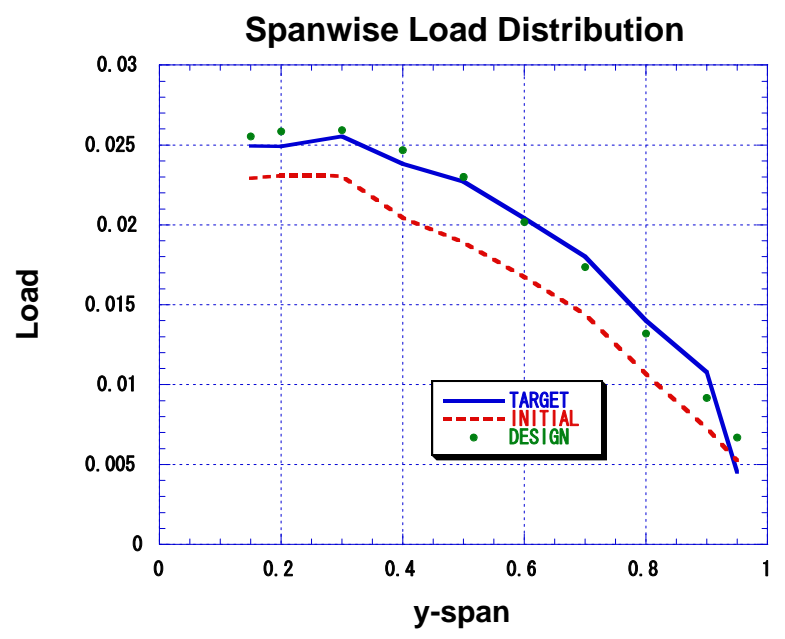

Fig. 7. Spanwise load distribution of wing-fuselage combination design problem 